\title{
Implications for first-order cosmological phase transitions and the formation of primordial black holes from the third LIGO-Virgo observing run
}

\section{A. Romero-Rodríguez ${ }^{a, *}$}

${ }^{a}$ Institut de Fisica d'Altes Energies (IFAE), Barcelona Institute of Science and Technology (BIST) Campus UAB, Facultat Ciencies Nord, 08193 Bellaterra, Barcelona, Spain

E-mail: aromero@ifae.es

We place constraints on the normalised energy density in gravitational waves from first-order strong phase transitions and from the formation of primordial black holes from curvature fluctuations using data from Advanced LIGO and Virgo's first, second and third observing runs. First, adopting a broken power law model, we place $95 \%$ confidence level upper limits simultaneously on the gravitational-wave energy density at $25 \mathrm{~Hz}$ from unresolved compact binary mergers and strong first-order phase transitions. We then consider two more complex phenomenological models, limiting at $25 \mathrm{~Hz}$ the gravitational-wave background due to bubble collisions and the background due to sound waves at $95 \%$ confidence level for phase transitions occurring at temperatures above $10^{8} \mathrm{GeV}$. Finally, we perform a similar search assuming a background sourced by the formation of primordial black holes and unresolved compact binary mergers. For a very generic spectrum describing the primordial black hole background, we place $95 \%$ confidence level upper limits on the gravitational-wave energy density at $25 \mathrm{~Hz}$

*** The European Physical Society Conference on High Energy Physics (EPS-HEP2021), ***

*** 26-30 July $2021 * * *$

*** Online conference, jointly organized by Universität Hamburg and the research center DESY ***

\footnotetext{
${ }^{*}$ Speaker
} 


\section{Stochastic gravitational wave background from Phase Transitions}

In a First Order Phase Transition (FOPT), it is well established that gravitational waves (GWs) can be produced by mainly three sources: bubble collisions, sound waves, and magnetohydrodynamic turbulence [1]. The GW thus produced is a stochastic gravitational wave background (SGWB), described by the energy density spectrum: $\Omega_{\mathrm{gw}}(f)=d \rho_{\mathrm{gw}} /\left(\rho_{\mathrm{c}} d \ln f\right)$ with $\rho_{c}$ the present critical energy density $\rho_{\mathrm{c}}=3 c^{2} H_{0}^{2} /(8 \pi G), \rho_{\mathrm{gw}}$ the density of GW and $f$ the frequency. Each spectrum can be well approximated by a broken power law, with its peak frequency determined by the typical length scale at the transition, the mean bubble separation $R_{\mathrm{pt}}$ which is related to the inverse time duration of the transition $\beta$, and also by the amount of redshifting determined by $T_{\mathrm{pt}}$ and the cosmic history. The amplitude of each contribution is largely determined by the energy released normalized by the radiation energy density $\alpha$, its fraction going into the corresponding source and the bubble wall velocity $v_{\mathrm{w}}$. The dominant source for GW production in a thermal transition, as most commonly encountered in the early universe, is the sound waves in the plasma induced by the coupling between the scalar field and the thermal bath [2]. We use the spectrum from numerical simulations:

$\Omega_{\mathrm{sw}}(f) h^{2}=2.65 \times 10^{-6}\left(\frac{H_{\mathrm{pt}}}{\beta}\right)\left(\frac{\kappa_{\mathrm{sw}} \alpha}{1+\alpha}\right)^{2}\left(\frac{100}{g_{*}}\right)^{1 / 3} \times v_{\mathrm{w}}\left(\frac{f}{f_{\mathrm{sw}}}\right)^{3}\left(\frac{7}{4+3\left(f / f_{\mathrm{sw}}\right)^{2}}\right)^{7 / 2} \Upsilon\left(\tau_{\mathrm{sw}}\right)$,

where $\kappa_{\mathrm{sw}}$ is the fraction of vacuum energy converted into the kinetic energy of the bulk flow, a function of $v_{\mathrm{w}}$ and $\alpha$ [3]; $H_{\mathrm{pt}}$ is the Hubble parameter at $T_{\mathrm{pt}} ; g_{*}$ is the number of relativistic degrees of freedom, chosen to be 100 in our analysis; $h$ is the dimensionless Hubble parameter; $f_{\mathrm{sw}}$ is the present peak frequency, $f_{\mathrm{sw}}=19 \frac{1}{v_{\mathrm{w}}}\left(\frac{\beta}{H_{\mathrm{pt}}}\right)\left(\frac{T_{\mathrm{pt}}}{100 \mathrm{GeV}}\right)\left(\frac{g_{*}}{100}\right)^{\frac{1}{6}} \mu \mathrm{Hz}$, and $\Upsilon=1-\left(1+2 \tau_{\mathrm{sw}} H_{\mathrm{pt}}\right)^{-1 / 2}$ which is a suppression factor due to the finite lifetime, $\tau_{\mathrm{SW}}$, of sound waves. When sound waves, and thus also magnetohydrodynamic turbulence, are highly suppressed or absent, bubble collisions can become dominant, e.g., for a FOPT in vacuum of a dark sector which has no or very weak interactions with the standard plasma. The resulting GW spectrum can be well modelled with the envelope approximation [4], which assumes infinitely thin bubble wall and neglects the contribution from overlapping bubble segments. In the low-frequency regime, $\Omega_{\mathrm{gw}} \propto f^{3}$ from causality [5], and for high-frequencies $\Omega_{\mathrm{gw}} \propto f^{-1}$ due to the dominant single bubble contribution as revealed by the analytical calculation [6]. The spectrum is [6]:

$$
\Omega_{\mathrm{coll}}(f) h^{2}=1.67 \times 10^{-5} \Delta\left(\frac{H_{\mathrm{pt}}}{\beta}\right)^{2}\left(\frac{\kappa_{\phi} \alpha}{1+\alpha}\right)^{2} \times\left(\frac{100}{g_{*}}\right)^{1 / 3} S_{\mathrm{env}}(f),
$$

where $\kappa_{\phi}=\rho_{\phi} / \rho_{\text {vac }}$ denotes the fraction of vacuum energy converted into gradient energy of the scalar field. The amplitude $\Delta$ is $\Delta\left(v_{\mathrm{w}}\right)=0.48 v_{\mathrm{w}}^{3} /\left(1+5.3 v_{\mathrm{w}}^{2}+5 v_{\mathrm{w}}^{4}\right)$ and the spectral shape is $S_{\text {env }}=1 /\left(c_{l} \tilde{f}^{-3}+\left(1-c_{l}-c_{h}\right) \tilde{f}^{-1}+c_{h} \tilde{f}\right)$ where $c_{l}=0.064, c_{h}=0.48$ and $\tilde{f}=f / f_{\text {env }}$ with $f_{\text {env }}$ the present peak frequency: $f_{\mathrm{env}}=16.5\left(\frac{f_{\mathrm{bc}}}{\beta}\right)\left(\frac{\beta}{H_{\mathrm{pt}}}\right)\left(\frac{T_{\mathrm{pt}}}{100 \mathrm{GeV}}\right)\left(\frac{g_{*}}{100}\right)^{\frac{1}{6}} \mu \mathrm{Hz}$, and $f_{\mathrm{bc}}$ the peak frequency right after the transition $f_{\mathrm{bc}}=0.35 \beta /\left(1+0.069 v_{\mathrm{w}}+0.69 v_{\mathrm{w}}^{4}\right)$.

\subsection{Bayesian analysis}

We follow V. Mandic et al. approach [7, 8] to perform a Bayesian search and model selection. We analyse the O1-O3 LIGO-Virgo data, for which we perform a multi-baseline study summing 
the corresponding log-likelihoods for individual pairs of detectors. We undertake a study on simultaneous estimation of a Compact Binary Coalescence (CBC) background and a background sourced by FOPTs, because current estimates of the CBC background [9] show it as a non-negligible component of any SGWB signal. The CBC spectrum is modelled as $\Omega_{\mathrm{cbc}}=\Omega_{\mathrm{ref}}\left(f / f_{\mathrm{ref}}\right)^{2 / 3}$, with $f_{\text {ref }}=25 \mathrm{~Hz}$ as the reference frequency. The search for an isotropic stochastic signal shows no evidence of correlated magnetic noise, and a pure Gaussian noise model is still preferred by the data [9]. Therefore, here, a contribution from Schumann resonances [10] is neglected.

\subsection{Data analysis and results}

We take two analysis approaches. First, we consider an approximated broken power law including main features of the shape and its peak (see Ref.[11] for the expression of the energy density spectrum, the priors used in the search as well as the results). We then consider the phenomenological models (Eqs. (1) and (2)), for contributions from bubble collisions and sound waves.

We follow an approach similar to that by B. Von Harling et al. [12]. The parameters of the search are $\boldsymbol{\theta}_{\mathrm{gw}}=\left(\Omega_{\mathrm{ref}}, \alpha, \beta / H_{\mathrm{pt}}, T_{\mathrm{pt}}\right)$ including CBC background $\Omega_{\mathrm{cbc}}$, and $\Omega_{\mathrm{gw}}$ from bubble collisions and sound waves described by Eqs. (2) and (1), respectively. For bubble collisions, $v_{\mathrm{w}}$ and $\kappa_{\phi}$ are set to unity. The remaining model parameters are varied in the ranges specified in Ref. [11]. For sound waves, we initially set $v_{\mathrm{w}}=1$, and then explore different values for $v_{\mathrm{w}}$ in the range $(0.7$ 1.0 ), corresponding to various detonation and hybrid modes of fluid velocity profile [3]. The rest of the parameters are varied as in the case of bubble collisions. We now estimate 95\% CL upper limits on $\Omega_{\text {coll }}$ and $\Omega_{\mathrm{sw}}$ with priors stated in Ref. [11]. We obtained Bayes factors $\log \mathcal{B}_{\text {noise }}^{\text {cbc }+ \text { coll }}=$ -0.74 and $\log \mathcal{B}_{\text {noise }}^{\text {cbc }+ \text { ww }}=-0.66$, for bubble collisions and sound waves, respectively. In Fig. 1(a) we present exclusion regions as a function of the different parameters of the CBC+FOPT model, now under the assumption that contributions from bubble collisions dominate, with $v_{\mathrm{w}}=1$ and $\kappa_{\phi}=1$. In general, with the chosen prior, the data can exclude part of the parameter space at $95 \% \mathrm{CL}$, especially when $T_{\mathrm{pt}}>10^{8} \mathrm{GeV}, \alpha>1$, or $\beta / H_{\mathrm{pt}}<1$. Similarly, in Fig. 1(b) we present the results for the CBC+FOPT hypothesis in which the sound waves dominate with $v_{\mathrm{w}}=1$ and $\kappa_{\mathrm{sw}}$ a function of $v_{\mathrm{w}}$ and $\alpha$. The Bayesian analysis shows sensitivity at large values of $\alpha$ and $T_{\mathrm{pt}}$, but does not exclude regions in the parameter space at $95 \% \mathrm{CL}$. The analysis is then performed for given values of $\beta / H_{\mathrm{pt}}$ and $T_{\mathrm{pt}}$ leaving $\alpha$ as a free parameter. As a result, a 95\% CL upper limit on $\Omega_{\mathrm{sw}}(25 \mathrm{~Hz})$ of $5.9 \times 10^{-9}$ is obtained for $\beta / H_{\mathrm{pt}}<1$ and $T_{\mathrm{pt}}>10^{8} \mathrm{GeV}$. The results indicate the importance of using LIGO-Virgo GW data to place constraints on new phenomena related to strong FOPTs in the early universe.

\section{Stochastic gravitational wave background from Primordial Black Holes}

Primordial Black Holes (PBHs) have gained interest in recent years as the traditional particle dark matter candidates have become increasingly tightly constrained. In this section we focus on a GW source related to PBHs, the large curvature fluctuations which lead to PBH formation. The most studied PBH formation mechanism relies on large curvature fluctuations generated during cosmological inflation [13]. The PBH formation is accompanied with a strong stochastic GW background generated at the second order in the cosmological perturbation theory from scalar perturbations [14]. The spectrum of the scalar induced GWs is fixed by the curvature power 


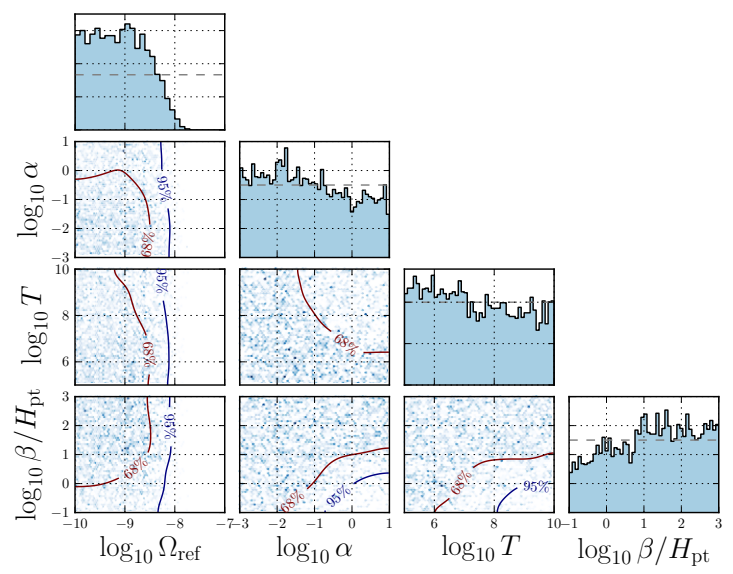

(a) Dominant bubble collision contributions as a function $\log _{10} \Omega_{\text {ref }}$ and the different parameters of the model.

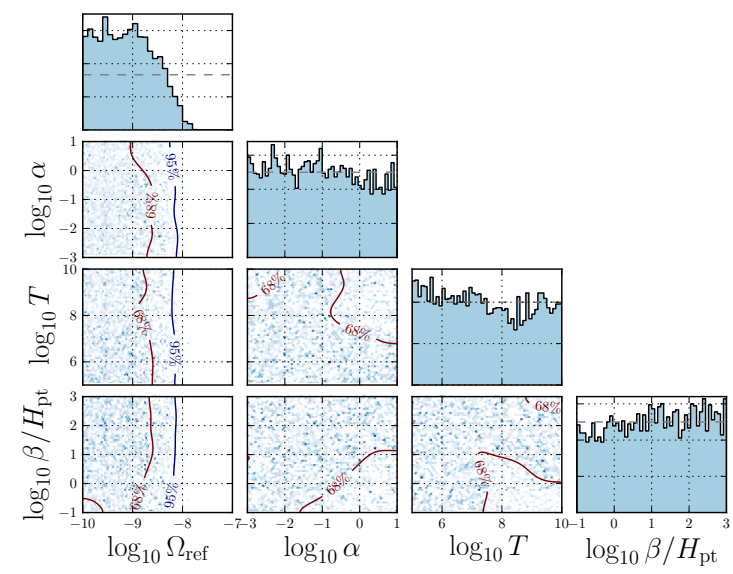

(b) Dominant sound wave contributions $\left(v_{\mathrm{w}}=1\right)$, as a function $\log _{10} \Omega_{\text {ref }}$ and the different parameters of the model.

Figure 1: Posterior distributions for the different CBC+FOPT searches. The $68 \%$ and $95 \%$ CL exclusion contours are shown. The horizontal dashed line in the posteriors indicates the priors used in the analysis.

spectrum (from the CMB observations we know that at large scales its amplitude is of $O\left(10^{-9}\right)$, which cannot be probed with any foreseeable experiment). For PBH formation the curvature power spectrum amplitude needs to be $O(0.01)$ at some small scales. This corresponds to a sizeable scalar induced GW background within the reach of GW observatories. Peaks in the curvature power spectrum that reach the amplitude $O(0.01)$ required for $\mathrm{PBH}$ formation can be generated by features or turns in the inflaton potential. Nevertheless, we will not consider any particular model of inflation. Instead, we parametrize the peak in the curvature power spectrum by its position $\left(k_{*}\right)$, width $(\Delta)$ and integrated power $(A)$ :

$$
\mathcal{P}_{\zeta}(k)=\frac{A}{\sqrt{2 \pi} \Delta} \exp \left[-\frac{\ln ^{2}\left(k / k_{*}\right)}{2 \Delta^{2}}\right]
$$

The expression for the scalar induced GW spectrum in terms of the curvature power spectrum is given in Refs. [14]. This spectrum is peaked around the same wavenumber as the curvature power spectrum, corresponding to the frequency $f_{*} / \mathrm{Hz}=1.6 \times 10^{-15} k_{*} / \mathrm{Mpc}^{-1}$, and its peak amplitude is $\Omega_{\mathrm{GW}}=O\left(10^{-5}\right) A^{2}$ in the $\Delta \ll 1$ limit. The LIGO-Virgo detectors, being sensitive to frequencies around $f \gtrsim 10 \mathrm{~Hz}$, have the potential to probe peaks in the curvature power spectrum at scales larger than $10^{15} \mathrm{Mpc}^{-1}$. Such scales re-entered the horizon at very high temperatures, $T \gtrsim 10^{8} \mathrm{GeV}$, so, assuming the Standard Model value for the effective number of relativistic degrees of freedom, we take $g_{*, S}=g_{*} \approx 100$. We perform a search of a scalar induced GW background in the LIGO-Virgo data generated by such peaks in the curvature power spectrum, and study whether LIGO-Virgo observations constrain the aforementioned PBH formation scenario.

\subsection{Data analysis and results}

Similarly to what is described in section 1.1, we perform a simultaneous Bayesian search for a stochastic GW signal sourced by both scalar fluctuations and CBCs. The data is fitted to a model 
$\Omega_{\mathrm{GW}}(f, \boldsymbol{\theta})$ that includes four parameters, $\boldsymbol{\theta}=\left(\Omega_{\mathrm{ref}}, A, k_{*}, \Delta\right)$. The priors used for each of the variables can be found in Ref. [15].

The results from the Bayesian search and the posterior distributions of the different parameters are in Fig. 2. A Bayes factor of $\ln \mathcal{B}_{\text {noise }}^{\mathrm{CBC}+\text { scalar }}=-0.8$ is obtained, indicating no evidence for a stochastic signal, as described by the combined $\mathrm{CBC}$ and scalar induced GW model, in the LIGO-Virgo data. A $95 \%$ confidence level (CL) upper limit on $\Omega_{\mathrm{CBC}}(25 \mathrm{~Hz})=6.0 \times$ $10^{-9}$ was obtained, compatible with the results from other searches [11]. The data excludes part of the parameter space in $k_{*} / \mathrm{Mpc}^{-1}$ and $A$, which shows significant dependence on $\Delta$.

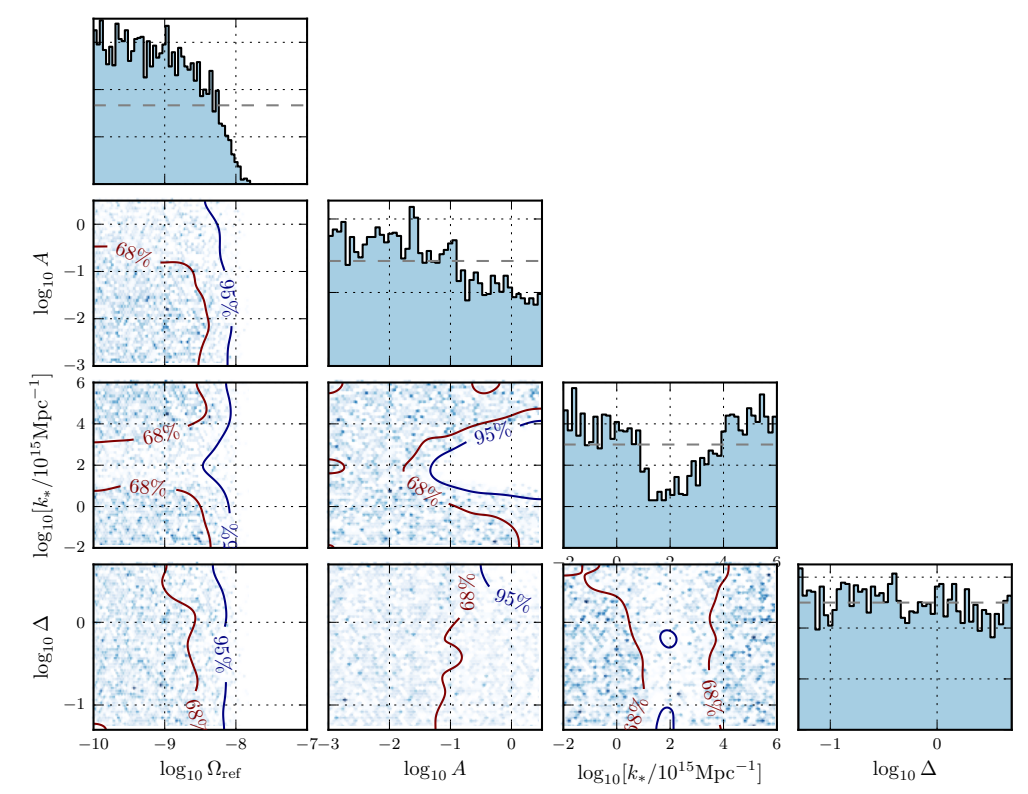

Figure 2: Posterior distributions for the combined $\mathrm{CBC}$ and scalar induced GW search. The $68 \%$ and $95 \%$ CL exclusion contours are shown. The horizontal dashed lines in the histograms indicate the priors.

\subsection{Implications}

In Fig. 3 we show the current 95\% CL LIGO-Virgo bound for the peak integrated power $A$ of the curvature power spectrum as a function of the peak wavenumber $k_{*}$ obtained from the Bayesian analysis for a Dirac delta function peak $(\Delta \rightarrow 0)$ and for a log-normal peak with $\Delta=1$. In the range $O\left(10^{15}\right)<k_{*} / \mathrm{Mpc}^{-1}<O\left(10^{18}\right)$ the LIGO-Virgo bound is stronger than the indirect bounds on the abundance of GWs arising from big bang nucleosynthesis (BBN), $\Omega_{\mathrm{GW}} h^{2}<1.8 \times 10^{-6}$ [14], and the cosmic microwave background (CMB) observations, $\Omega_{\mathrm{GW}} h^{2}<1.7 \times 10^{-6}$ [16]. To compare the LIGO-Virgo bound on $A$ to the constraints induced by PBH formation, we calculate the PBH abundance following the calculation presented in Ref. [17] of the PBH formation. In Fig. 3 we show the envelope of the collection of these constraints by the green curves. As discussed above, we consider two cases which reflect the theoretical uncertainties in the PBH formation. The upper green curve corresponds to $\kappa=3.0$ and $\delta_{c}=0.65$, and the lower one to $\kappa=11.0$ and $\delta_{c}=0.45$ (see the definition of these parameters in Ref. [15]). We see that the current 95\% CL LIGO-Virgo bound lies above both of these curves, and therefore we conclude that currently still the LIGO-Virgo sensitivity is not enough to constrain the PBH formation. The dashed contours instead show the projected exclusion for Advanced LIGO at its designed sensitivity and that of the next generation experiment Einstein telescope (ET). These are calculated by requiring a signal-to-noise ratio for signal detection above eight, and indicate that in near future the ground-based GW detectors could probe PBH formation. 

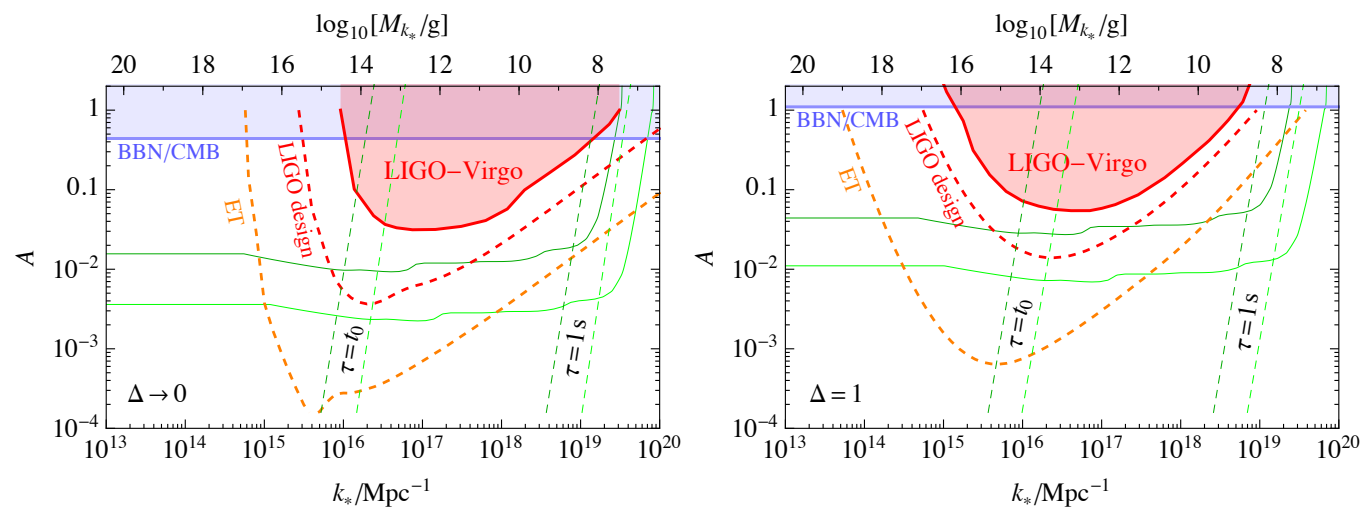

Figure 3: Comparison between the LIGO-Virgo 95\% CL exclusions (red solid lines) for $\Delta \rightarrow 0$ (left) and $\Delta=1$ (right) and the constraints arising from BBN/CMB (blue lines) and PBH formation on the integrated power of the curvature power spectrum (see text). The dashed red and orange curves show the projected sensitivities of the LIGO in its final phase and Einstein telescope (ET).

\section{References}

[1] R.-G.Cai, Z. Cao, Z.-K. Guo, S.-J.Wang and T.Yang (2017), arXiv: 1703.00187.

[2] M. Hindmarsh, et. al. Phys. Rev. Vol.D92, (2015), 1504.03291.

[3] J. R. Espinosa et al., JCAP Vol.06, (2010), 1004.4187.

[4] A. Kosowsky and M. S.Turner, Phys.Rev Vol.D47, (1993), astro-ph/9211004.

[5] M. Maggiore, Gravitational Waves. Vol. 2: Astrophysics and Cosmology , 2018).

[6] R.Jinno and M.Takimoto, Phys. Rev. Vol.D95, (2017), 1605.01403.

[7] V. Mandic, E. Thrane, S. Giampanis, T. Regimbau, Phys. Rev. Lett. Vol.109, (2012),

[8] T. Callister, et. al. PhysRevX. Vol.7, (2017)1704.08373.

[9] B. Abbott et al. (LIGO-Virgo Scientific Collaboration), LIGO-DCC:P2000314 (2020b),

[10] E. Thrane, N. Christensen, R. Schofield, Phys. Rev. D Vol.87, (2013),

[11] A.Romero, et. al.. Phys.Rev.Lett. Vol.126 (2021)

[12] B. Von Harling, A. Pomarol, O. Pujolàs, and F. Rompineve, JHEP Vol.04, (2020), 1912.07587.

[13] S. Hawking, Mon. Not. Roy. Astron. Soc. Vol.152, (1971).

[14] K. Kohri, T. Terada, PhysRevD. Vol.97, (2018), 1804.08577.

[15] A.Romero, et al. (2021) arXiv:2009.05514 [gr-qc]

[16] L. Pagano, et. al. Phys. Lett. B Vol.760, (2016)1508.02393.

[17] S. Young, I. Musco, C.T. Byrnes, JCAP Vol.11, (2019)1904.00984. 